\title{
Torsional Deformations in Subnanometer MoS Interconnecting Wires
}

\author{
Ai Leen Koh, ${ }^{\dagger}$ Shanshan Wang, ${ }^{\ddagger}$ Can Ataca, ${ }^{\S}$ Jeffrey C. Grossman, ${ }^{\S}$ Robert Sinclair, ${ }^{\prime}$ \\ and Jamie H. Warner*, \\ ${ }^{\dagger}$ Stanford Nano Shared Facilities, Stanford University, Stanford, California 94305-4045, United States \\ ${ }^{\ddagger}$ Department of Materials, University of Oxford, Parks Road, Oxford, OX1 3PH, United Kingdom \\ ${ }^{\S}$ Department of Materials Science and Engineering, Massachusetts Institute of Technology, 77 Massachusetts Avenue, Cambridge, \\ Massachusetts 02139-4307, United States \\ "Department of Materials Science and Engineering, Stanford University, Stanford, California 94305-4034, United States
}

\section{Supporting Information}

\begin{abstract}
We use aberration-corrected transmission electron microscopy to track the real time atomic level torsional dynamics of subnanometer wires of MoS interconnecting monolayer regions of $\mathrm{MoS}_{2}$. An in situ heating holder is used inside the transmission electron microscope to raise the temperature of the sample to $400{ }^{\circ} \mathrm{C}$ to increase crystallization rates of the wires and reduce contamination effects. Frequent rotational twisting of the MoS wire is captured, demonstrating elastic torsional deformation of the MoS wires. We show that torsional rotations of the crystal structure of the MoS wires depend upon the specific atomic structure of the anchored sections of the suspended wire and the number of unit cells that make up the wire length. Elastic torsional flexibility of the MoS wires is revealed to help their self-adapting connectivity during the structural changes. Plastic torsional deformation is also seen for MoS wires that contain defects in their crystal structure, which produce small scale rotational disorder within the wires. Upon removal of the defects, the wire returns back to pristine form. These results provide detailed insights into how the atomic structure of the anchoring site significantly influences the nanowire configurations relative to the monolayered $\mathrm{MoS}_{2}$.
\end{abstract}

KEYWORDS: MoS wire, nanowire, TEM, DFT, aberration-corrected

$\mathrm{N}$ anowires are one-dimensional (1D) structures that have promise in applications ranging from transistor electronics to interconnects. Obtaining suspended subnanometer wires is quite challenging, as they are often unstable and fragile. Carbon nanotubes have long been studied as an ideal form of 1D carbon structures, along with metal wires produced by solution phase methods. ${ }^{1,2}$ There has also been an extensive amount of research done on filling the interior of carbon nanotubes with inorganic material to achieve template growth of $1 \mathrm{D}$ nanowires, but removing the carbon housing has been a major challenge. ${ }^{3-7}$ With the emergence of $2 \mathrm{D}$ materials, there is interest in the development of interconnecting nanowires that are strongly bonded to the edge of the $2 \mathrm{D}$ sheets, rather than simply weakly adhered to the surface.

Recent work has revealed that subnanometer wires interconnecting domains of monolayer transition metal dichalcogenides (i.e., $\mathrm{MoS}_{2}$ and $\mathrm{MoSe}_{2}$ ) can form from electron beam irradiation. ${ }^{8,9}$ Nanowires in monolayer TMDs can be created by electron beam induced sputtering of the $S$ atoms or Se atoms and creating holes in the material. When the holes meet, the nanowires form with $\mathrm{MoS}$ stoichiometry for $\mathrm{MoS}_{2}$ monolayer starting material. ${ }^{8,9}$ Recent reports showed these subnanometer wires are flexible, self-adapting, and can rotate their entire crystal structure under electron beam irradiation. ${ }^{8}$ Figure 1 shows such an example of this behavior, observed in our samples. The rotation of the entire MoS crystal has been demonstrated to be associated with changes in the anchoring attachment to the $\mathrm{MoS}_{2}$ monolayer 2D material. This gives rise to two main projections and phase contrast patterns in highresolution transmission electron microscopy (HRTEM): the well-defined contrast spots with alternating zigzag geometry, as in Figure 1a,c, and then the rotated projection in Figure $1 \mathrm{~b}, \mathrm{~d}$ that results in blurring of the lattice spots perpendicular to the wire's long axis to give equally spaced lines of contrast. Understanding this relationship between crystal projection and phase contrast pattern enables an examination of fluctuations along the nanowire length and exploration of torsional effects such as the rotation of the crystal structure and defect-induced lattice rotation.

Highly anisotropic crystal structures, nanowires, are prone to rotational distortions, such as the helical twists in cobalt iodide

Received: November 5, 2015

Revised: December 18, 2015

Published: January 19, 2016 


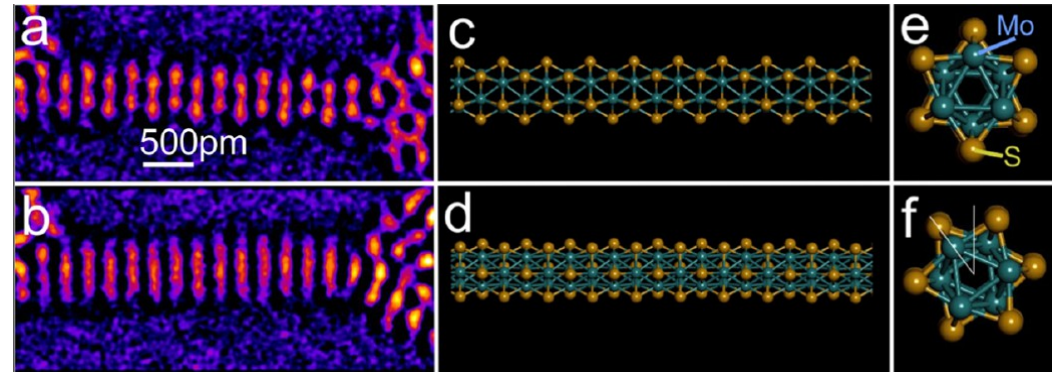

Figure 1. Projection-dependent phase contrast images of $\mathrm{MoS}$ wires. (a,b) AC-TEM images of a suspended MoS wire in monolayer $\mathrm{MoS}_{2}$ before (a) and after (b) a whole rotation about its long axis. Time between images is $1 \mathrm{~s}$. (c,d) Schematic atomic models indicating the projection of the MoS crystal structure after a rotation, where (c) corresponds to the AC-TEM image in (a) and (d) corresponds to the AC-TEM image in (b). (e,f) End views of the atomic models in $(c, d)$, respectively, showing the relative orientation of the entire MoS nanowire structure about its long axis. White lines in (f) show the relative orientations between the two crystals.

wires formed within carbon nanotubes that were imaged at the atomic scale. ${ }^{10}$ A total crystal rotation (helical twist) of $\sim 50^{\circ}$ was observed over a distance of $\sim 10 \mathrm{~nm}$, giving a rotation of $\sim 5^{\circ} / \mathrm{nm}$. A similar helical chiral twist was reported for $2 \mathrm{D}$ closed packed fullerene chains inside carbon nanotubes, where helical twists of $90^{\circ}$ were observed over $\sim 26 \mathrm{~nm}$, giving $\sim 3.5^{\circ} \%$ $\mathrm{nm}$ rotation in the crystal structure. ${ }^{11}$ In both these cases, the interaction with the carbon nanotube and its flexibility as a host container is an influencing factor on the final structural dynamics of the system. In larger nanowire systems, such as $\mathrm{PbSe}$, Eshelby twists of $45^{\circ}$ over a length of $9 \mu \mathrm{m}$ were reported, giving a rotation of $\sim 0.005^{\circ} / \mathrm{nm}$. ${ }^{12}$ While there has been some theoretical analysis of the atomic structure of nanowires during torsional deformations, there has been little experimental work on probing the rotational twists, such as torsional effects, on the crystal structures of suspended nanowires with subnanometer widths. ${ }^{13-15}$ In particular, when a suspended wire is rigidly clamped at both ends by strong covalent bonding it provides constraints to the rotational freedom that influence the torsional strain.

Here, we show that significant torsional effects are present in 1D MoS nanowires that are related to the structure of the anchoring attachment sites to the $2 \mathrm{D}$ material. The rotation of the crystal structure is driven by changes to the attachment of one end of the suspended MoS wire or by changes in the number of unit cells that make up the wire length, relative to the anchoring attachment sites. These results reveal important insights related to the competing process of shearing and crystal rotation to compensate for induced torsional strain in the system. Monolayer $\mathrm{MoS}_{2}$ was grown by chemical vapor deposition using methods similar to those previously reported and transferred to a holey SiN TEM grid. ${ }^{16}$ We examined areas of $\mathrm{MoS}_{2}$ free from carbon residue to eliminate its effect on nanowire formation. An FEI Environmental Titan XFEG TEM at an accelerating voltage of $80 \mathrm{kV}$ was used to image the sample in high vacuum at up to 10 frames per second using a Gatan OneView camera. Samples were heated to $400{ }^{\circ} \mathrm{C}$ during image using an in situ heating holder. This helped to reduce contamination effects and also increase the rate of crystallization of the MoS nanowires compared to room temperature.

Results and Discussion. MoS wires were formed by constant electron beam irradiation of the monolayer $\mathrm{MoS}_{2}$ sample using an $80 \mathrm{keV}$ electron beam, similar to previous reports. ${ }^{8,9}$ The atomic structure of the wires we observed match the structure reported in ref 8 . Once the MoS wires formed, they are stable for sufficient time to enable more than 1000 frames to be acquired over several minutes. We used an in situ
TEM heating holder set to $400{ }^{\circ} \mathrm{C}$ that we found increases the yield of crystalline $\mathrm{MoS}$ nanowire production compared to room-temperature experiments. Figure 2 shows a series of

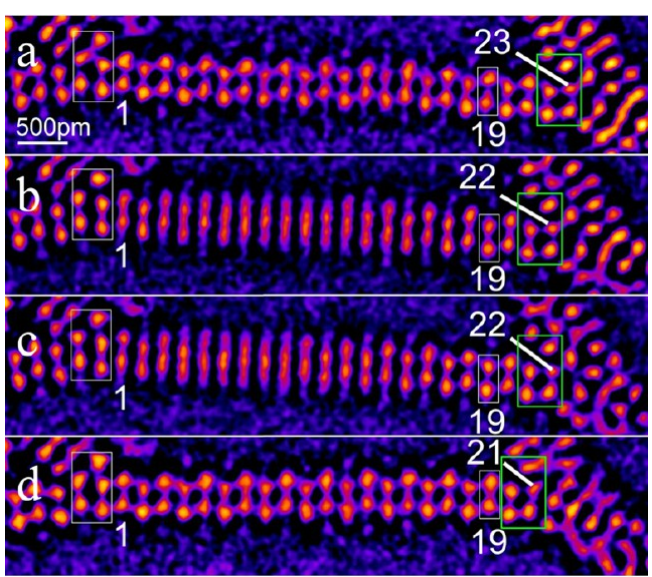

Figure 2. (a-d) Series of AC-TEM images as a function of time, showing torsional rotation of MoS wire. Rotated regions seen as blurred sections of wire in $(b, c)$. White box on the left shows the anchoring site that is constant through-out the series of images. Numbers indicate the relative lattice plane segments relative to the left anchor site. The right anchor site is indicated with the green box and the white line shows its number relative to the left anchor site. White box on the right highlights section 19 of the wire and its relative rotation to section 1 . Time between frames $\sim 1 \mathrm{~s}$.

aberration-corrected TEM (AC-TEM) images of a MoS wire undergoing structural transformation. In Figure 2a, clear contrast spots are observed for the wire, indicating a crystal projection similar to that described in Figure 1a. The left anchor site of the wire is indicated with a white box and remains fixed throughout the image series. The right anchor site indicated with the white line also remains relatively fixed throughout the image series, however the number of segments of lattice that comprise the wire's length change throughout the sequence. The wire contains 23 segments between the anchoring points in Figure 2a, but in the next frame (Figure $2 b$ ) one segment is lost, effectively shortening the wire. Given that the anchoring structure at both ends remain constant (white and green boxes), it indicates that the segment is lost from the middle region of the wire. Segment number 19 in Figure $2 b, c$, as counted from the left anchor point, shows a $60^{\circ}$ phase shift (i.e., rotation) relative to segment 19 in Figure 2 a. The blurred lattice structure in Figure $2 b$ indicates a rotation of 


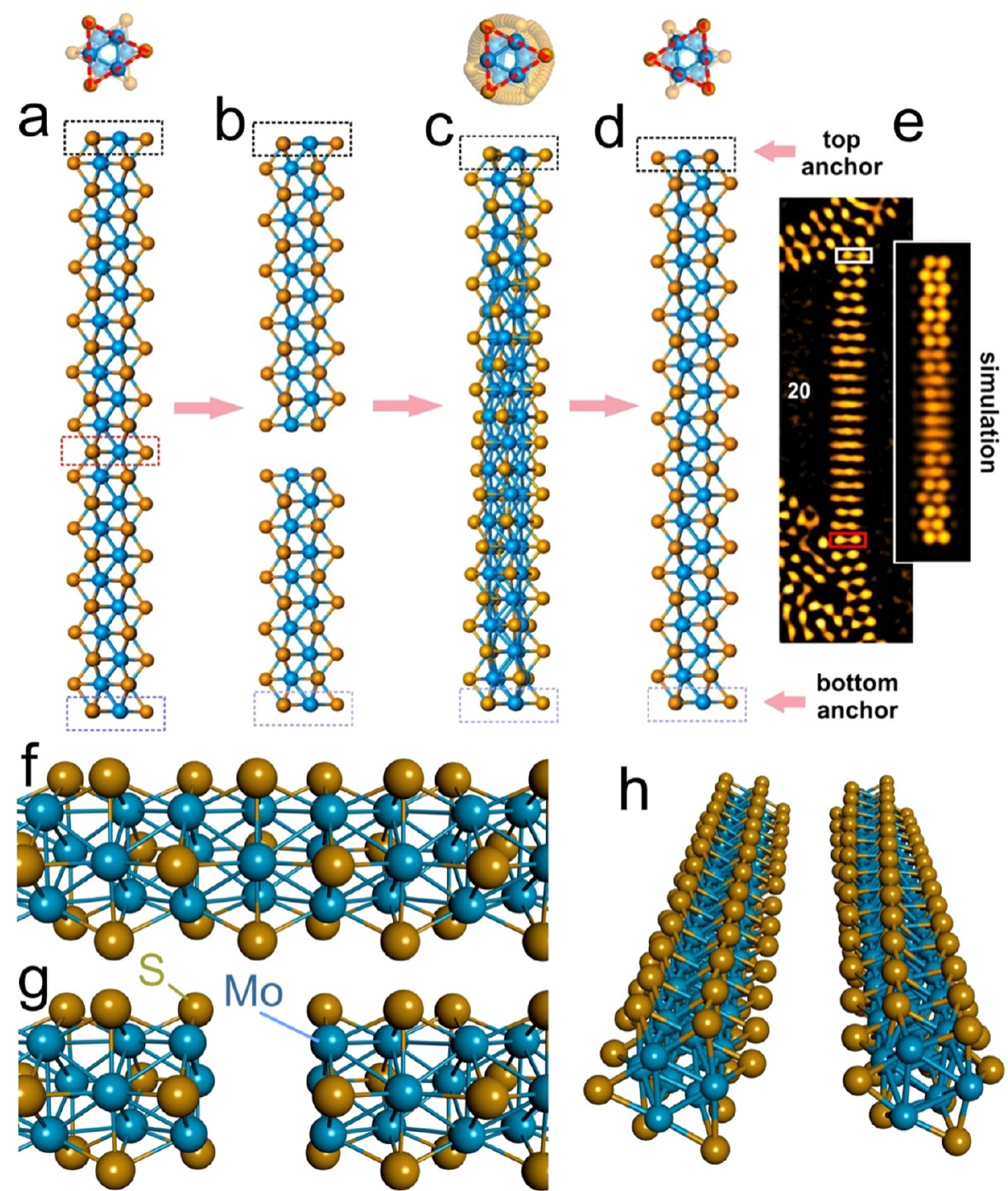

Figure 3. $(a-d)$ Schematic atomic models showing how rotational twists are introduced into MoS wires by the loss of one lattice segment. (a) Pristine MoS wire. (b) One segment removed from middle section. (c) Rotational twisting of MoS wire after bond reconstruction with fixed anchor points. (d) Rotation of top anchor point resulting in antitwisting of MoS wire and return to nontwisted form. (e) AC-TEM image of twisted wire and multislice image simulation based on the atomic model in (c). (f) A 3D perspective view of MoS atomic model of pristine wire. (g) After the removal of one segment exposing the identical faces either side. (h) A 3D perspective view of pristine and twisted MoS wires.

the lattice projection, as mentioned in Figure 1d. This structure is maintained in Figure 2c, confirming it is a stable structural form of the wire. The anchor points at both ends in Figure $2 b, c$ shows no signs of rotation and this reveals that there is torsional rotation within the wire structure, which is explained in more detail in Figure 3. In Figure 2d, another segment is lost, reducing the length of the wire again, and this eliminates the torsional rotation of the lattice and the wire returns back to the same orientation that in which it started. Segment 19 in Figure $2 \mathrm{~d}$ has also undergone a second $60^{\circ}$ phase shift to return back to its original orientation. This behavior is repeated in many suspended wire structures, where losing one segment causes torsional rotation in the wire, provided that no changes to the anchoring points occurs.

The schematic atomic models in Figure 3 describe the mechanisms by which the torsional rotation is induced. From Figure 3, panel a to b, one segment is lost, and this leaves the two opposing segments to have identical orientation, which is likely to be unstable due to further bond reconstruction. The loss of a segment in the nanowire is likely due to the effect of compression strain induced on the wire as the two anchoring ends come closer together during the electron beam irradiation process that causes vibrations of the entire sample region. Some sputtering of atoms by the electron beam may also occur, causing loss of a segment in the nanowire. Figure $3 \mathrm{f}$,g shows 3D perspective models of the MoS nanowire with and without the segment removed, exposing the two identical faces that reconstruct to induce lattice rotation. With both ends of the wire fixed, the structure can easily relax by small scale segmentby-segment crystal rotation to form the twisted structure in Figure $3 c$. This structure can revert back to nontorsional state by the rotation of its anchor end, as in Figure $3 \mathrm{~d}$, essentially eliminating the mismatch. Figure $3 e$ shows an AC-TEM image of such a twisted MoS wire with a multislice image simulation shown next to it obtained from the atomic model presented in Figure $3 \mathrm{c}$. The blurring of the lattice structure is observed in the middle region of the simulation, confirming the experimental analysis. Figure $3 \mathrm{~h}$ shows $3 \mathrm{D}$ perspective views 
of the original and twisted MoS wire. The total angle twisted along the length of the wire should stay the same, $60^{\circ}$, in order to achieve such structures. Therefore, the degree of rotation per nanometer will depend upon the length of wire. Longer wires will have smaller degree of rotation per nanometer compared to shorter wires and a higher degree of rotation per nanometer requires greater strain on the bonds between each segment due to the deviation from perfect symmetry of the crystal structure. At some point, the wire will become too short for it to be energetically favorable to adopt rotational twists, and instead it is likely that shearing effects will cause defects in the lattice structure to occur. Theoretical simulations showed that MoS nanowires are stable to torsions up to $67^{\circ} / \mathrm{nm}$.

In Figure 4, we use density functional theory (DFT) to examine the length-dependent energy of rotational twist within the $\mathrm{MoS}$ wire. Figure $4 \mathrm{a}$ shows the pristine $\mathrm{MoS}$ wire segmented into 12 primitive unit cells that are repeated to give the arbitrary length. Figure $4 \mathrm{e}$ shows how the torsion
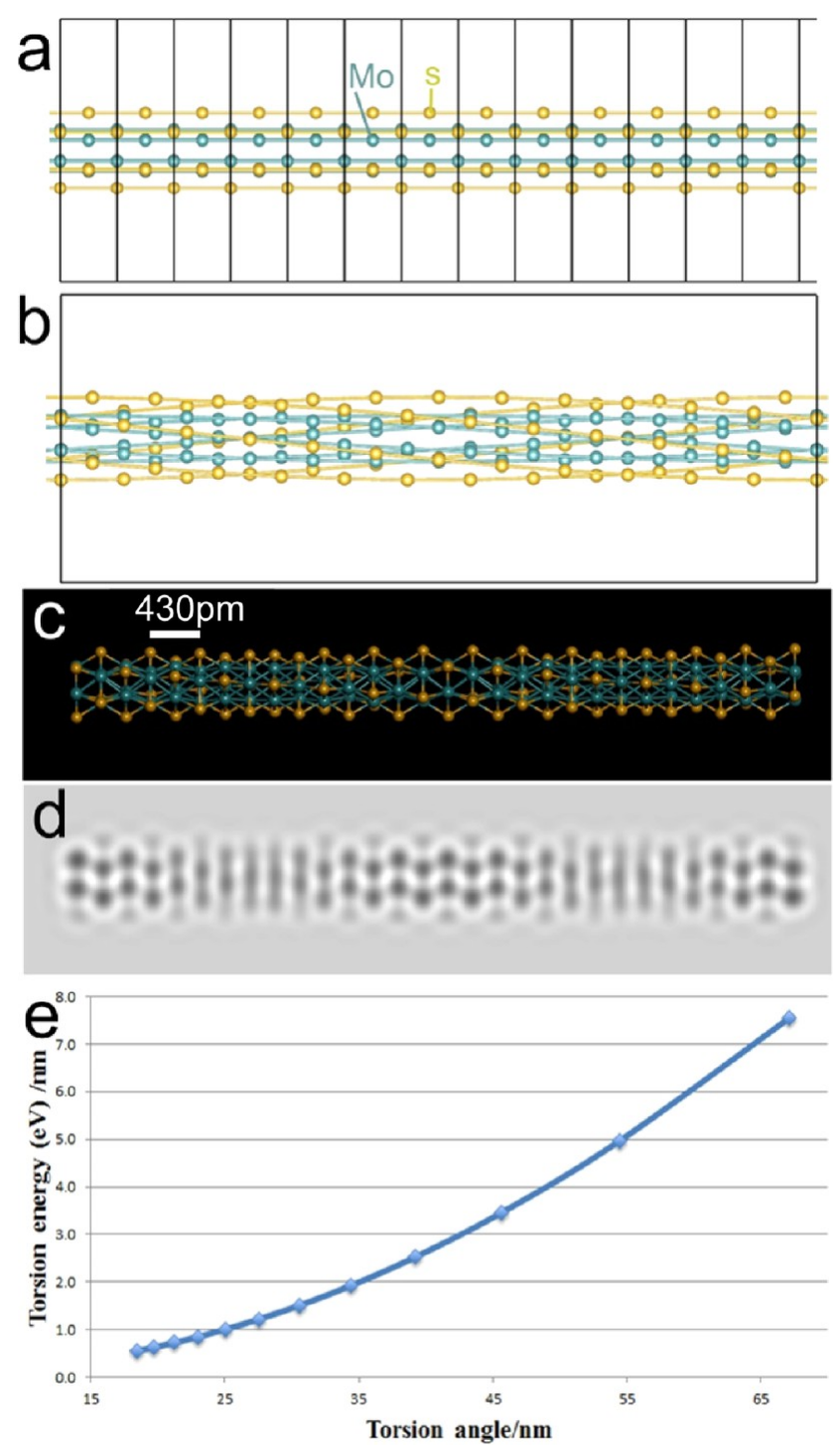

Figure 4. (a) DFT relaxed atomic structure of (a) pristine MoS wire and (b) MoS wire twisted $120^{\circ}$. Primitive unit cell is indicated (c) with DFT relaxed atomic model used for the multislice image simulation shown below in (d). (e) Plot of torsion energy per $\mathrm{nm}$ as a function of the torsion angle per $\mathrm{nm}$. energy decreases as the length of the MoS wire is increased. The $x$-axis shows the number of repeating primitive unit cells over which the $120^{\circ}$ rotation occurs, which is related to the rotation angle per nanometer for a wire. As expected, the torsion energy decreases substantially as the length of wire increases. Figure $4 \mathrm{c}$ shows a DFT relaxed atomic model of a MoS wire rotated over $120^{\circ}$, and the multislice image simulation is shown below in Figure 4d. The image simulation shows strong contrast spots when the angle of rotation equals 0,60 , and $120^{\circ}$ and blurred lines for all other torsion angles, agreeing with the experimental results. Band structure calculations from the DFT for torsion angle/nanometers ranging from 18 to $67^{\circ}$ show that the torsional wires retain their metallic behavior when twisted, which is important for their possible functionality as interconnects.

Figure 5 shows a slightly shorter wire than the one examined in Figure 2, where defects in the lattice are seen to occur as the length of the wire is reduced. In Figure 5a, the wire is pristine, and by the end in Figure $5 j$ one segment of the wire is lost. Figure $5 \mathrm{~b}$ shows the emergence of a defect in the middle region of the wire, it has the form of an edge dislocation, where a row of atoms is missing. This causes the section of the wire to the right of the defect to be blurred, likely due to rotation of the crystal lattice by a fixed amount. This is also seen again in Figure 5e. The defect propagates along the wire to the left with some shearing of the wire observed in Figure 5c. Reconstruction occurs between Figure 5d,f, leaving an extra Mo atom on the edge of the wire, Figure $5 \mathrm{~g}$. There is also a lattice mismatch and phase inversion of the segment stacking in the wire, highlighted by the white brackets in Figure 5f. By Figure $5 \mathfrak{j}$, the defect in the MoS wire has been eliminated and the wire structure returns to the same form as it originally had in Figure 5a.

Figure 6 shows another example of MoS nanowire twisting occurring. Figure 6a shows a well-ordered MoS wire with sharp contrast spots and segment 19 is indicated as the segment that forms the anchor to the right side. The left anchor site remains constant throughout the image sequence, indicated with the white box in Figure 6. In the next frame, Figure $6 \mathrm{~b}$, the right attachment site of the wire reconstructs and segment 20 now becomes the anchor site on the right. The growth of the wire by one segment causes little rotational distortion to the wire because the right anchor site adapts to enable the elongation. Again in the next frame, Figure $6 c$, the wire grows by one segment, and now segment 21 becomes the right anchor site. Almost no rotation is observed in the wire growth due to the anchor reconstruction that enables the growth. From Figure 6, panel $\mathrm{f}$ to $\mathrm{g}$, one segment is lost, and two distinct sections of the wire are apparent in both panels $g$ and h of Figure 6 where the left side shows sharp contrast spots and the right side shows blurred lines. This defect is observed at the interface of these two sections and is the likely source of the abrupt lattice rotation. The defect is eliminated by Figure 6i, resulting in a more continuous torsional rotational of the lattice, seen by blurring of contrast spots in segment 6 between Figure 6 panels $\mathrm{h}$ and i. Finally, from Figure 6, panel i to j, the right anchor point reconstructs and the wire increases in length by one segment and the rotation of the lattice is eliminated. These images show how the MoS wire self-adapts to the anchoring point at the edge during length changes by defect formation and elimination, lattice rotations, followed by anchor site reconstruction. 


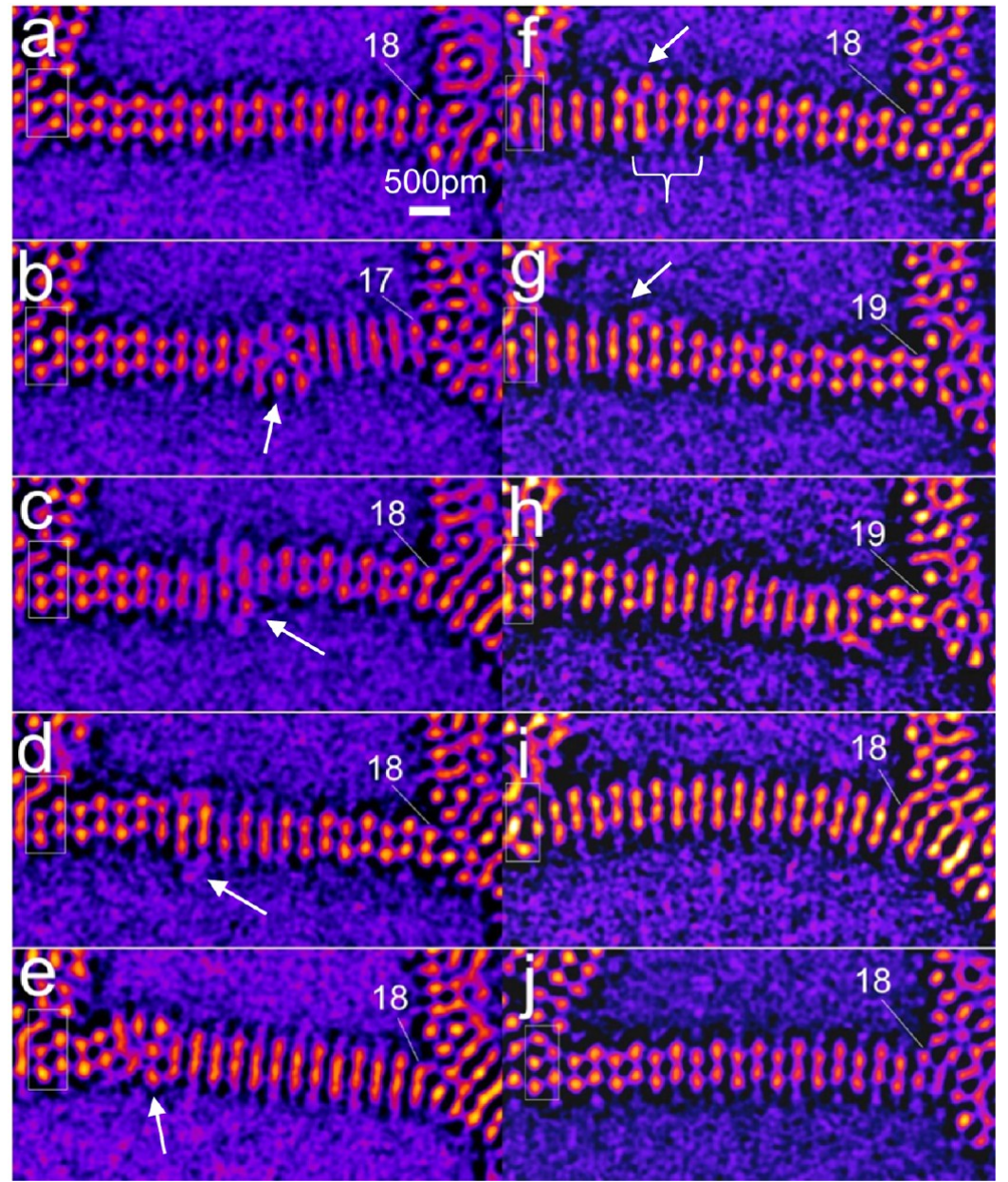

Figure 5. Series of AC-TEM images showing defect propagation in MoS wires. Time between frames is $\sim 0.5 \mathrm{~s}$.

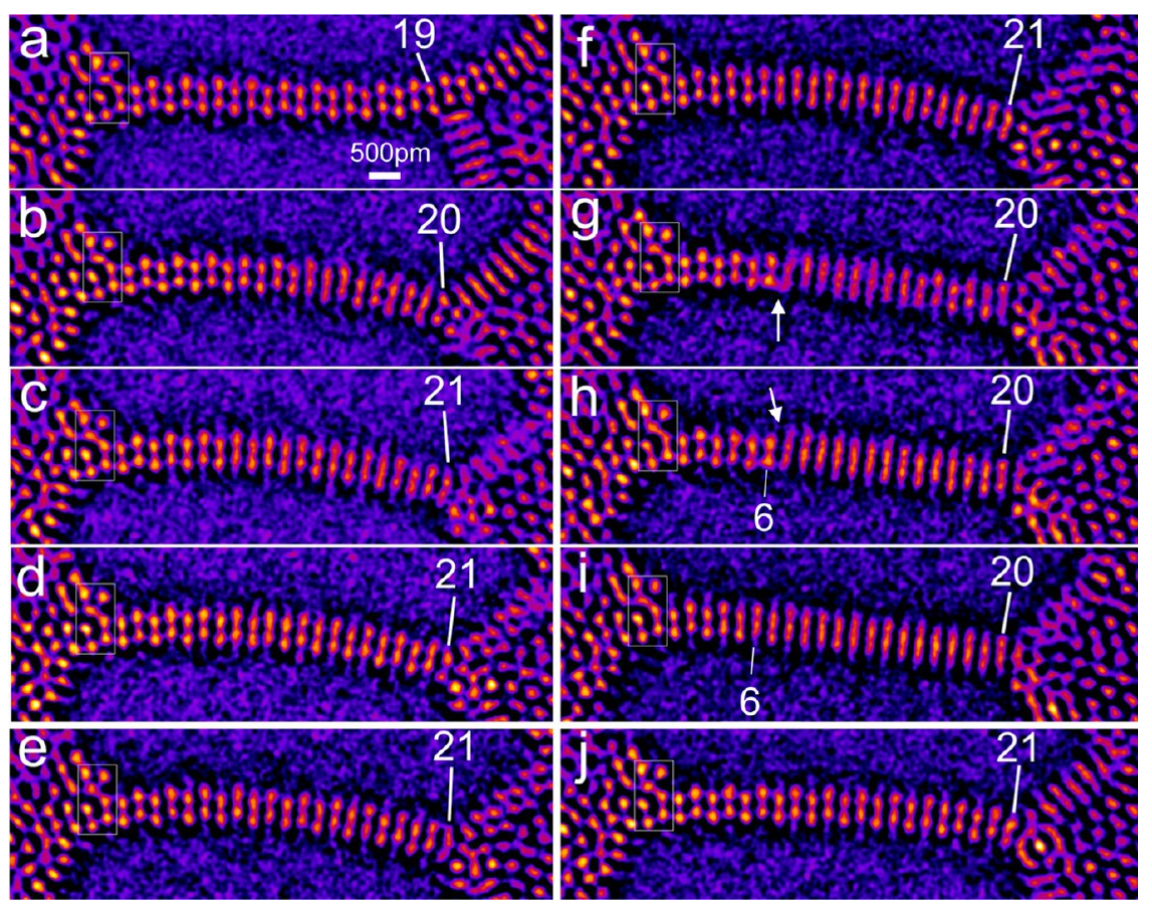

Figure 6. $(a-j)$ Series of AC-TEM images showing the dynamics of a MoS wire during reconstruction. Time between frames $\sim 1 \mathrm{~s}$. 
During the observation of the MoS nanowire by AC-TEM the anchoring points of the $\mathrm{MoS}_{2}$ suspended wire are prone to beam induced movements. This can have the effect of increasing the distance between the two anchor points of the $\mathrm{MoS}$ wire, sometimes snapping it or decreasing the distance, which is often the instigator for the torsional twists as the wire seeks to accommodate the strain induced from compression. The MoS wires are often seen to bend, indicating there is some flexure, but ejection of material from the central region of the wire seems to be a frequent occurrence when length contraction is required. As previously mentioned, the loss of a segment in the MoS wire induces torsional lattice effects that are relieved by either further loss of another segment or by readjustment of the anchoring sites.

We found that this behavior was not just limited to the MoS wires that interconnect $\mathrm{MoS}_{2}$ domains but also can be seen in $\mathrm{MoS}$ wires that form at the edge of $\mathrm{MoS}_{2}$ during electron beam irradiation. Figure 7 shows a series of AC-TEM images of a

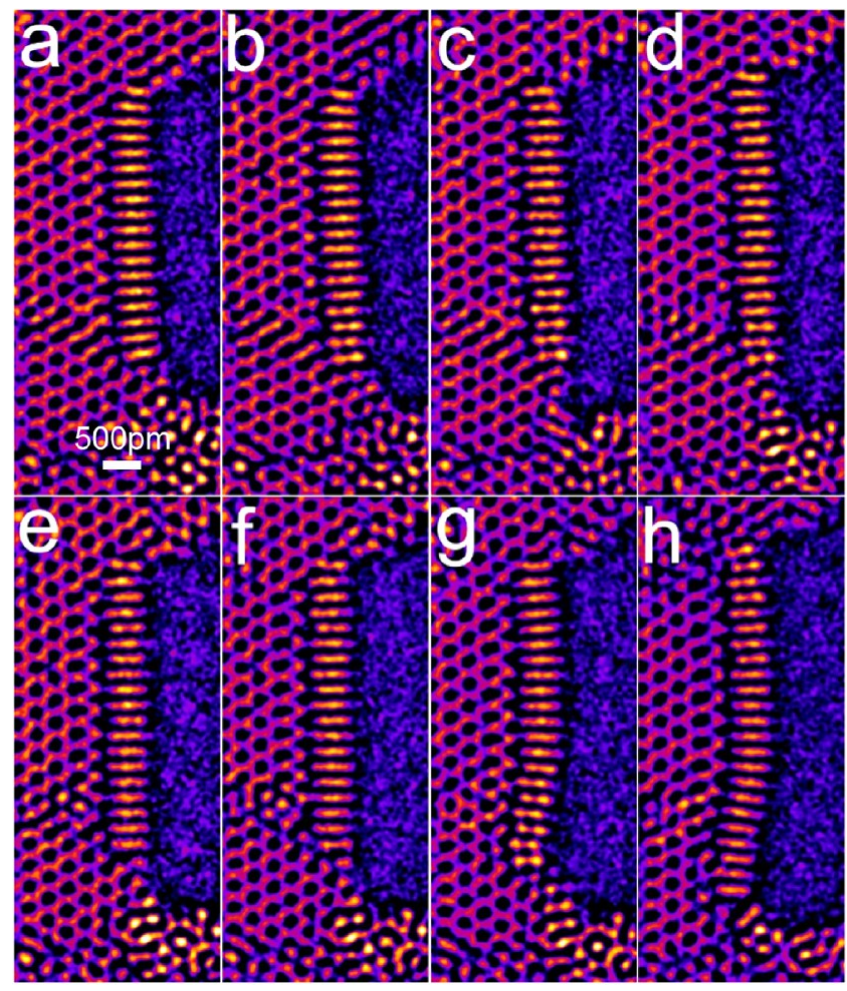

Figure 7. $(\mathrm{a}-\mathrm{h})$ Series of AC-TEM images showing the dynamics of a $\mathrm{MoS}$ wire located at the edge of monolayer $\mathrm{MoS}_{2}$. Time between frames is $\sim 0.5 \mathrm{~s}$.

$\mathrm{MoS}$ wire at the zigzag edge of $\mathrm{MoS}_{2}$. Wires at the edge also show either sharp contrast spots or blurred lines as described in Figure 1. In Figure 7a, the wire shows blurred contrast across the entire length. In the next frame, Figure $7 b$, the bottom anchor site has reconstructed to show sharp contrast spots, which remain in Figure $7 c, d$. In Figure $7 e$, this anchor site reverts back and shows blurred contrast in Figure $7 \mathrm{f}$ as well. By Figure $7 \mathrm{~g}$, a large portion of the bottom section of the MoS wire has rotated to show sharp contrast spots again with the top section showing blurred lines. This again is indicative of torsional twists in the MoS wire along its length. In the next frame, Figure $7 \mathrm{~h}$, both anchor ends have adjusted with the top section now showing sharp contrast spots and the bottom section showing blurred line contrast. In Figure $7 \mathrm{~g}, \mathrm{~h}$, the nanowire also shows bending. These results show that torsional rotation of wires can also occur for MoS along the edges of $\mathrm{MoS}_{2}$. Careful examination of the periodicity of the $\mathrm{MoS}_{2}$ zigzag edge sites with the positions of contrast of the MoS wire show no real correlation, indicating that the main bonding occurs at the ends of the wire and not in the main body segment. This also supports the idea that the rotation of the wire is driven by changes to the end anchor groups, because bonding within the main wire body to the edge would prohibit wire rotation.

Conclusion. Studying the dynamics of $\mathrm{MoS}$ wires in $\mathrm{MoS}_{2}$ monolayers showed unique torsional rotation effects that help the self-adapting nature during growth and contraction of the wire. Using a fast frame rate camera with high sensitivity aids in acquiring dynamics that reveal changes in the MoS structure. Losing a segment of lattice in MoS wires induces rotation of the crystal structure, which is then relieved by the reconstruction of one of the anchor sites. This reveals that these subnanometer $\mathrm{MoS}$ interconnecting wires exhibit both bending flexure and rotational "elastic" torsion. In some cases, plastic deformation of the MoS wire by the generation of defects helps to relieve the torsional strain. These defects migrate along the wire before being eliminated and the wire reverts back to pristine form through atomic reconstruction of anchor sites.

Method. Synthesis and Transfer of Monolayer $\mathrm{MoS}_{2}$. $\mathrm{MoS}_{2}$ monolayers were grown using a hydrogen-free chemical vapor deposition method in atmospheric pressure with precursors of molybdenum trioxide $\left(\mathrm{MoO}_{3}, \geq 99.5 \%\right.$, SigmaAldrich) and sulfur ( $S, \geq 99.5 \%$, Sigma-Aldrich) powder, similar to a previously reported strategy. ${ }^{16}$ The $\mathrm{MoO}_{3}$ monolayers were grown on the $\mathrm{SiO}_{2} / \mathrm{Si}\left(300 \mathrm{~nm}\right.$ thick $\left.\mathrm{SiO}_{2}\right)$. To avoid the cross-contamination between $\mathrm{MoO}_{3}$ and $\mathrm{S}$ powder at high temperature $\mathrm{MoO}_{3}$ precursor was initially loaded in a smaller diameter tube of around $1 \mathrm{~cm}$, which was then placed into the larger 1 in. quartz tube for the entire CVD growth, while the $S$ powder was put in the outer tube. The mouth of the inner tube, which is located near the gas inlet, was positioned upstream more than $15 \mathrm{~cm}$ from the position of $S$, which can thoroughly avoid the $S$ vapor to spread into the inner tube and react with $\mathrm{MoO}_{3}$. Two furnaces were applied to provide a better temperature control for both precursors and the substrate. $S$ powder and the substrate (with face up) were placed in the central area of the first and second furnace, respectively, while $\mathrm{MoO}_{3}$ powder was loaded at the upstream of the second furnace. The heating temperature for $\mathrm{S}, \mathrm{MoO}_{3}$, and substrate were $\sim 180, \sim 300$, and $\sim 800{ }^{\circ} \mathrm{C}$ with argon used as the carrier gas. After a preintroduction of $S$ vapor for $\sim 15 \mathrm{~min}$, the temperature for the second furnace was first increased to $\sim 800{ }^{\circ} \mathrm{C}$ at a ramping rate of $40{ }^{\circ} \mathrm{C} / \mathrm{min}$ and maintained for 15 min under 150 standard cubic centimeters per minute $(\mathrm{sccm})$ argon flow. Next, the argon flow was reduced to $10 \mathrm{sccm}$ and kept for $25 \mathrm{~min}$ before the growth stopped, followed by a fast cooling process. The temperature for $S$ was retained at $\sim 180$ ${ }^{\circ} \mathrm{C}$ during the whole synthesis period. After growth, the surface of the $\mathrm{MoS}_{2} / \mathrm{SiO}_{2} / \mathrm{Si}$ substrate was first spin-coated with a thin film of poly(methyl methacrylate) (PMMA) followed by floating it on a $1 \mathrm{~mol} / \mathrm{L}$ potassium hydroxide solution to etch $\mathrm{SiO}_{2}$ away. After it peeled off, the PMMA/ $\mathrm{MoS}_{2}$ film was then transferred into the deionized water for several times to wash off any residual contamination from the etching process. The rinsed $\mathrm{PMMA} / \mathrm{MoS}_{2}$ film was subsequently scooped up by a holey $\mathrm{Si}_{3} \mathrm{~N}_{4}$ TEM grid, (Agar Scientific Y5358), air-dried overnight, and baked at $180{ }^{\circ} \mathrm{C}$ for $15 \mathrm{~min}$ to ensure a strong 
interface contact between $\mathrm{MoS}_{2}$ monolayers and the TEM grid. Finally, the PMMA scaffold was removed by submerging the TEM grid in the acetone solution for $8 \mathrm{~h}$.

Transmission Electron Microscopy with an in Situ Heating Holder. AC-TEM was conducted using an FEI Titan 80-300 environmental TEM at $80 \mathrm{kV}$ accelerating voltage with a Gatan OneView $(4 \mathrm{k} \times 4 \mathrm{k})$ high frame rate camera. In situ heating was achieved using a Gatan 652 Inconel heating holder. MoS nanowires were formed by exposing the monolayer $\mathrm{MoS}_{2}$ sample to the electron beam with the $80 \mathrm{kV}$ accelerating voltage. The sputtering of $S$ atoms causes holes to open up and eventually MoS wires are left interconnecting different regions of the sample. This process is similar to that reported in ref 9 .

Image Processing. Image J was used to process the AC-TEM images. A bandpass filter (between 100 and 1 pixels) and a Gaussian blur were carefully applied to minimize long-range uneven illumination without affecting the interpretation of the original image. A LUT of "fire" was used to improve visual inspection of TEM images. Multislice image simulations was accomplished using JEMS software with supercells created using Accelrys Discovery Studio Visualizer.

Density Functional Theory. First-principles DFT calculations were carried out using projector augmented wave potentials $^{17}$ and the generalized gradient approximation characterized by the Perdew-Burke-Ernzerhof ${ }^{18}$ exchangecorrelation functional with van der Waals corrections ${ }^{19}$ for both spin-polarized and spin-unpolarized cases. A large spacing of $\sim 20 \AA$ between wires was used in order to prevent interlayer interactions. A plane-wave basis set with kinetic energy cutoff of $300 \mathrm{eV}$ was used. In the self-consistent field potential and total energy calculations, the Brillouin zone was sampled by special $k$-points with a grid of $(51 \times 1 \times 1)$ for the primitive cell and scaled according for the size of the supercell. All atomic positions and lattice constants were optimized by using the conjugate gradient method, where the total energy was converged to $10^{-6} \mathrm{eV}$ between two consecutive steps, and the maximum Hellmann-Feynman forces acting on each atom was less than $0.01 \mathrm{eV} / \AA ̊$ upon ionic relaxation. The pressure in the unit cell was kept below $1 \mathrm{kBar}$ in the continuous lattice vector. Numerical calculations were performed using VASP. ${ }^{20}$

The primitive cell of the MoS wire contained two segments of triangles of $(\mathrm{MoS})_{3}$ with a torsion angle of $60^{\circ}$ such that rotating the primitive cell by $120^{\circ}$ returns back to itself. Simulations are carried out up to supercells containing 15 primitive cells (30 segments) for $120^{\circ}$ angle rotation. The torsion angle/nanometers is calculated as $120^{\circ} /$ length of supercell in nanometers. Torsion energy per nanometer is calculated as $\left(E_{\text {supercell }}-n^{*} E_{\text {primitive }}\right) /$ length of the supercell in $\mathrm{nm}$, where $E_{\text {supercell }}$ is the total energy of the supercell, which has $120^{\circ}$ torsion angle, and $E_{\text {primitive }}$ is the total energy of the primitive cell without any torsion.

\section{ASSOCIATED CONTENT}

\section{S Supporting Information}

The Supporting Information is available free of charge on the ACS Publications website at DOI: 10.1021/acs.nanolett.5b04507.

Video comprised of two sequential AC-TEM frames showing the mechanical compression of the $\mathrm{MoS}$ wire from local movements and the subsequent loss of material. (AVI)

\section{AUTHOR INFORMATION}

\section{Corresponding Author}

*E-mail: Jamie.warner@materials.ox.ac.uk.

\section{Notes}

The authors declare no competing financial interest.

\section{ACKNOWLEDGMENTS}

J.H.W. thanks the Royal Society for Support. Part of this work was performed at the Stanford Nano Shared Facilities.

\section{REFERENCES}

(1) Iijima, S.; Ichihashi, T. Single-Shell Carbon Nanotubes of $1 \mathrm{~nm}$ Diameter. Nature 1993, 363, 603-605.

(2) Huo, Z.; Tsung, C.-K.; Huang, W.; Zhang, X.; Yang, P. Sub-Two Nanometer Single Crystal Au Nanowires. Nano Lett. 2008, 8, 20412044.

(3) Sloan, J.; Kirkland, A. I.; Hutchison, J. L.; Green, M. L. H. Structural Characterization of Atomically Regulated Nanocrystals Formed within Single Walled Carbon Nanotubes Using Electron Microscopy. Acc. Chem. Res. 2002, 35, 1054-1062.

(4) Gautam, U. K.; Costa, P. M. F. J.; Bando, Y.; Fang, X.; Li, L.; Imura, M.; Goldberg, D. Recent Developments in Inorganically Filled Carbon Nanotubes: Successes and Challenges. Sci. Technol. Adv. Mater. 2010, 11, 054501.

(5) Liu, Z.; Suenaga, K.; Wang, Z.; Shi, Z.; Okunishi, E.; Iijima, S. Identification of Active Atomic Defects in a Monolayered Tungsten Disulphide Nanoribbon. Nat. Commun. 2011, 2, 213.

(6) Kitaura, R.; Imazu, N.; Kobayashi, K.; Shinohara, H. Fabrication of Metal Nanowires in Carbon Nanotubes via Versatile NanoTemplate Reaction. Nano Lett. 2008, 8, 693-699.

(7) Giusca, C. E.; Stolojan, V.; Sloan, J.; Borrnert, F.; Shiozawa, H.; Sader, K.; Rummeli, M. H.; Buchner, B.; Silva, S. R. P. Confined Crystals of the Smallest Phase Change Material. Nano Lett. 2013, 13, 4020-4027.

(8) Lin, J.; Cretu, O.; Zhou, W.; Suenaga, K.; Prasai, D.; Bolotin, K. I.; Cuong, N. Y.; Otani, M.; Okada, S.; Lupini, A. R.; Idrobo, J.-C.; Caudel, D.; Burger, A.; Ghimire, N. J.; Yan, J.; Mandrus, D. G.; Pennycook, S. J.; Pantelides, S. T. Flexible Metallic Nanowires with Self-Adaptive Contacts to Semiconducting Transition-Metal Dichalcogenide Monolayers. Nat. Nanotechnol. 2014, 9, 436-442.

(9) Liu, X.; Xu, T.; Wu, X.; Zhang, Z.; Yu, J.; Qiu, H.; Hong, J.-H.; Jin, C.-H.; Li, J.-X.; Wang, X.-R.; Sun, L.-T.; Guo, W. Top-Down Fabrication of Sub-Nanometre Semiconducting Nanoribbons Derived from Molybdenum Disulfide Sheets. Nat. Commun. 2013, 4, 1776.

(10) Philp, E.; Sloan, J.; Kirkland, A. I.; Meyer, R. R.; Friedrichs, S.; Hutchison, J. L.; Green, M. L. H. An Encapsulated Helical OneDimensional Cobalt Iodide Nanostructure. Nat. Mater. 2003, 2, 788791.

(11) Warner, J. H.; Wilson, M. Elastic Distortions of Carbon Nanotubes Induced by Chiral Fullerene Chains. ACS Nano 2010, 4, 4011-4016.

(12) Zhu, J.; Peng, H.; Marshall, A. F.; Barnett, D. M.; Nix, W. D.; Cui, Y. Formation of Chiral Branched Nanowires by the Eshelby Twist. Nat. Nanotechnol. 2008, 3, 477-481.

(13) Garcia, J. C.; Justo, J. F. Twisted Ultrathin Silicon Nanowires: A Possible Torsion Electromechanical Nanodevice. EPL 2014, 108, 36006.

(14) Cai, W.; Fong, W.; Elsen, E.; Weinberger, C. R. Torsion and Bending Periodic Boundary Conditions for Modeling the Intrinsic Strength of Nanowires. J. Mech. Phys. Solids 2008, 56, 3242-3258.

(15) Weinberger, C. R; Cai, W. Orientation-Dependent Plasticity in Metal Nanowires Under Torsion: Twist Boundary Formation and Eshelby Twist. Nano Lett. 2010, 10, 139-142.

(16) Wang, S.; Rong, Y.; Fan, Y.; Pacios, M.; Bhaskaran, H.; He, K.; Warner, J. H. Shape Evolution of Monolayer $\mathrm{MoS}_{2}$ Crystals Grown by Chemical Vapour Deposition. Chem. Mater. 2014, 26, 6371-6379. 
(17) Blöchl, P. E. Projector augmented-wave method. Phys. Rev. B: Condens. Matter Mater. Phys. 1994, 50, 17953-17979.

(18) Perdew, J. P.; Burke, K.; Ernzerhof, M. Generalized Gradient Approximation Made simple. Phys. Rev. Lett. 1996, 77, 3865-3868.

(19) Grimme, S. Semiempirical GGA-type Density Functional Constructed with a Long-range Dispersion Correction. J. Comput. Chem. 2006, 27, 1787.

(20) Kresse, G.; Hafner, J. Ab initio Molecular Dynamics for Liquid Metals. Phys. Rev. B: Condens. Matter Mater. Phys. 1993, 47, 558-561. 